\title{
Central pontine myelinolysis associated with acute haemorrhagic pancreatitis
}

\author{
RICHARD J. SHERINS AND M. ANTHONY VERITY \\ From the Departments of Medicine and Pathology, U.C.L.A. School of Medicine, \\ Los Angeles, California, U.S.A.
}

Acute pancreatitis is not only life-threatening in itself, but can also present a wide variety of complications that increase its morbidity. Particularly challenging are the extrapancreatic manifestations of the disease, which are rarely seen. Subcutaneous nodular fat necrosis (Weber-Christian syndrome), ascites, pleural effusions, nephrosis, fat emboli, venous thromboses and fat necrosis of bone have been documented recently (Lynch, 1954; Swerdlow, Berman, Gibbel, and Valaitis, 1960; Lucas and Owens, 1962).

Cerebral perivascular demyelination associated with acute haemorrhagic pancreatitis has been reported on one previous occasion (Vogel, 1951). However, other demyelinative encephalopathies have been described as a result of excessive alcohol ingestion and include the symmetrical demyelination of the corpus callosum described by Marchiafava and Bignami (1903), and central pontine myelinolysis recently described by Adams, Victor, and Mancall, (1959).

The case to be described is that of a middle-aged alcoholic male with relapsing pancreatitis who developed diffuse symmetrical demyelination of the pons and corpus callosum.

\section{CASE REPORT}

G. W., a 48-year-old Negro male, was admitted on 4 April 1965 because of severe abdominal pain of 20 hours' duration. He gave a history of excessive alcohol consumption for three weeks before admission. He was a heavy drinker for $\mathbf{2 5}$ years, imbibing as much as $\mathbf{3 0}$ fluid ounces per day. In 1951 he underwent an exploratory laparotomy for abdominal pain, and pancreatitits was found. In 1958 he was hospitalized for five days with a diagnosis of duodenal ulcer and acute pancreatitis which responded to conservative management. Twenty hours before admission the patient awoke with severe stabbing epigastric pain unrelieved by antacids. Jaundice, melaena, cramps, haematemesis, or an antecedent history of diabetes or steatorrhea were not present.

Upon admission, the patient was alert, but in pain, and appeared well-nourished with no loss of subcutaneous fat.
Temperature was $37.4^{\circ} \mathrm{C}$, pulse $115 / \mathrm{min}$ and blood pressure $145 / 95 \mathrm{~mm} \mathrm{Hg}$. Lipaemia retinalis was visualized. No cutaneous xanthomata were evident. All peripheral pulses were brisk. There was moderate guarding in the right upper quadrant of the abdomen and slight distention and rebound tenderness in the epigastrium. A neurological examination was unremarkable at this time and Chvostek's sign was absent. A diagnosis of acute pancreatitis was made. Laboratory findings included a haematocrit of $51 \%$, specific gravity of the urine was 1.019 , with no glucose present and $1+$ proteinuria, and negative urinary sediment. Serum creatinine concentration was $2.0 \mathrm{mg} \%$; serum amylase, 2,300 u.; cholesterol, 1,020 $\mathrm{mg} \%$; total lipids, $4,600 \mathrm{mg} \%$; triglycerides, $2,615 \mathrm{mg}$ $\%$; leucine aminopeptidase, 780 u.; SGOT 820 u.; SGPT, $270 \mathrm{u}$.; total bilirubin, $0.3 \mathrm{mg} \%$; calcium, $5.0 \mathrm{~m}$-equiv $/ 1$; blood glucose, $420 \mathrm{mg} \%$. Treatment included nasogastric suction, intravenous fluids, B-vitamins, atropine, meperidine, penicillin, and chlortetracycline.

Twelve hours after admission the patient became hypotensive, febrile, and very restless. A positive Chvostek's sign developed with a serum calcium of $4 \cdot 3$ m-equiv/1. and magnesium of 0.9 m-equiv/1. Calcium gluconate and magnesium sulphate were given with large volumes of plasma and saline fluid replacement. Regular insulin was begun and some improvement seen (see Fig. 1).

On the third day, confusion appeared and was thought to represent delirium tremens precipitated by recent alcohol withdrawal. By the fifth day serum amylase, lipids, and calcium had returned to normal. Serum creatinine, however, was still $2.0 \mathrm{mg} \%$ and blood glucose $450 \mathrm{mg} \%$, despite $50 \mathrm{u}$. insulin daily.

On the eighth day (Fig. 1), the patient deteriorated with onset of obtundity, fever to $39 \cdot 4^{\circ} \mathrm{C}$, migratory thrombophlebitis, jaundice with total bilirubin $2 \cdot 1 \mathrm{mg} \%$, and elevated serum sodium. On the 10th hospital day the patient's mental status could best be described as akinetic mutism. Analysis of the cerebrospinal fluid revealed nothing of note. Control of the hyperglycaemia and hypernatraemia required intensive efforts. Eight days later a chest radiograph showed wide-spread pulmonary infiltrates. Blood cultures grew Pseudomonas and Polymyxin was added to the regimen. On the 35th hospital day, the patient died.

Necropsy was performed nine hours after death. The major visceral findings included severe acute and chronic haemorrhagic pancreatitis with necrosis and calcification 
of peripancreatic fat (Fig. 2); fibrous adhesions to stomach and duodenum; chronic gastric ulceration; haemorrhagic broncho-pneumonia and tracheitis; pulmonary oedema; early portal cirrhosis, and left ventricular hypertrophy.

The cerebral leptomeninges were thin and delicate after fixation in formalin. There was no evidence of increased intracranial pressure or gyral atrophy. There was minimal atherosclerosis, but no occlusive vascular lesions.
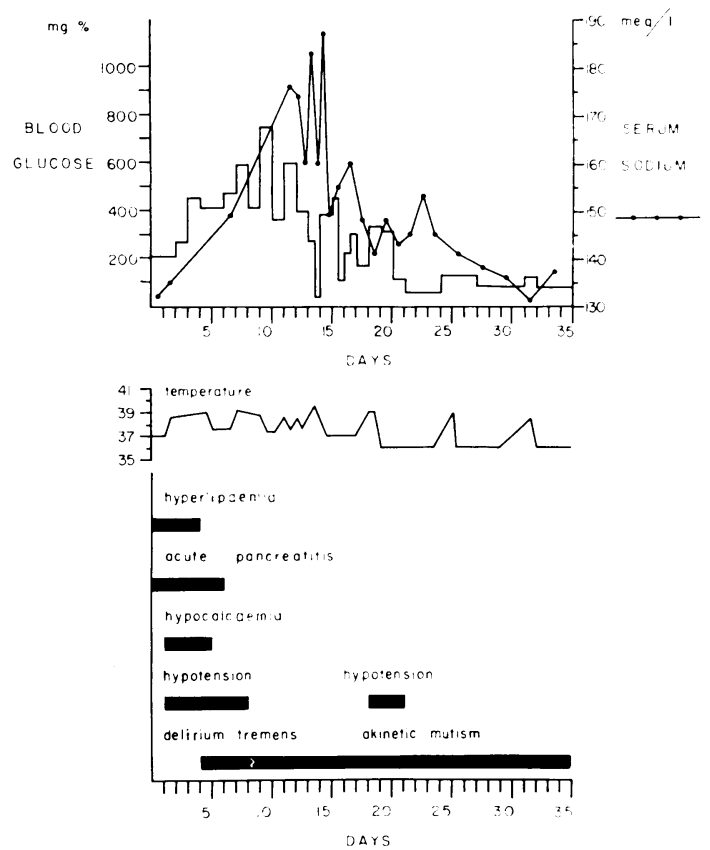

FIG. 1. Schematic summary of clinical course.

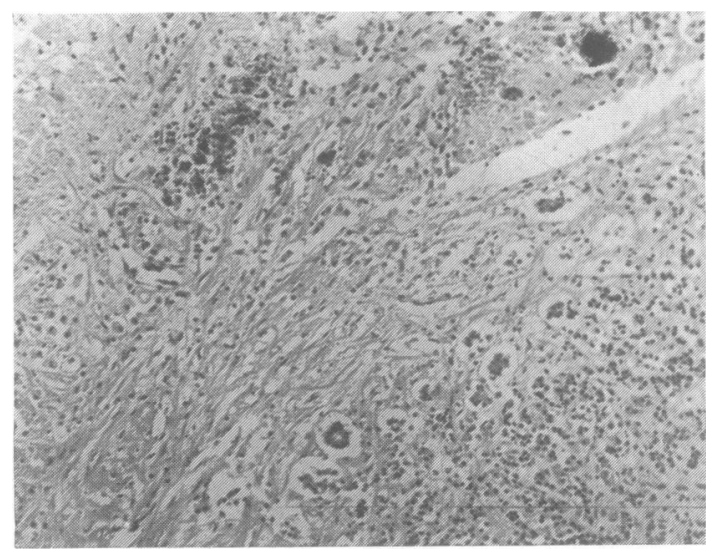

FIG. 2. Destruction of acinar architecture, extensive fibrosis, focal calcification, and amorphous peripheral necrosis. Section of pancreas $($ H.E. $\times 70)$.
After overnight storage in $80 \%$ ethanol, coronal sections revealed a central area of softening in the basilar portion of the pons with distortion of the transverse fibres and longitudinal fasciculi. Examination of coronal sections of the cerebral hemispheres displayed similar areas of irregular softening in the corpus callosum and medullary substance of the cerebellar hemispheres.

Microscopically, the areas of softening in the nervous system showed regions of demyelination, most extensive in the pons (Fig. 3). The pontine lesion was symmetrical, with central demyelination through the middle and lower portion. It involved the medial lemniscus bilaterally, but

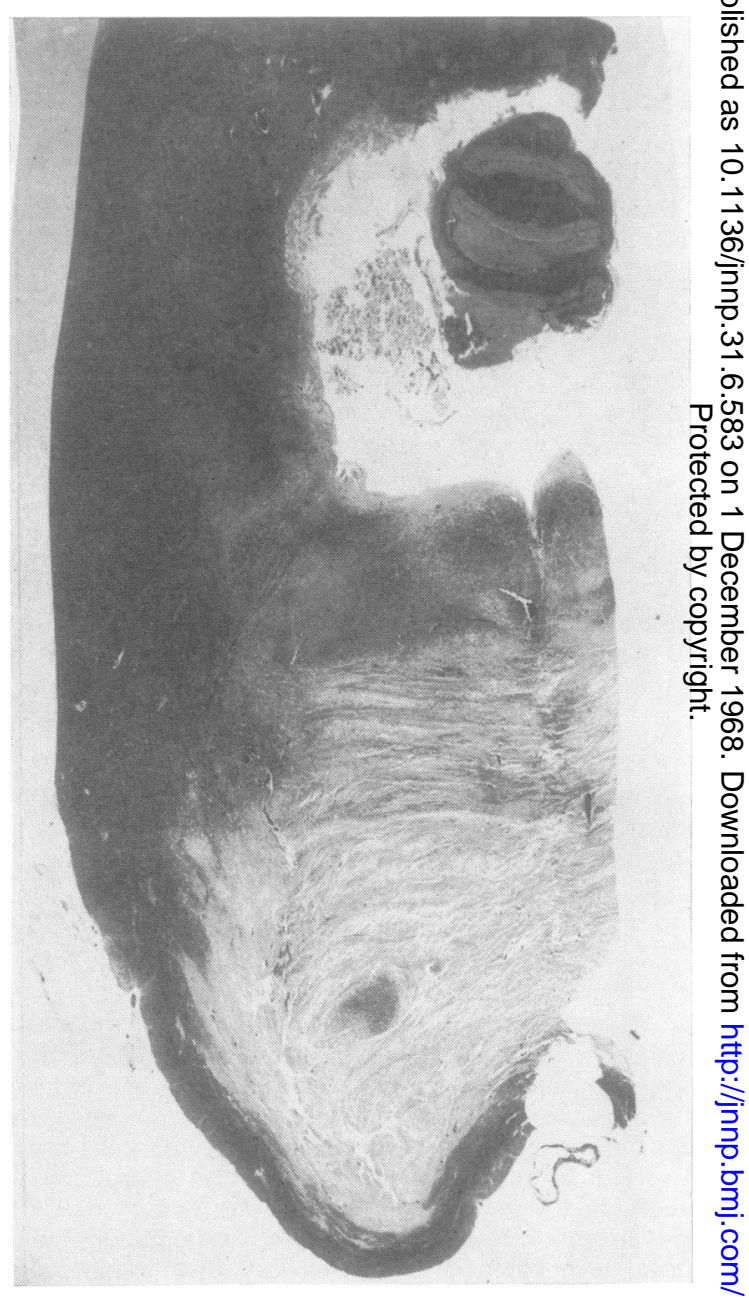

FIG. 3. Hemi-coronal section of pons immediately caudal 윽 to origin of trigeminal nerve. Extensive demyelination of the pons extends to the trapezoid body and medial lemniscus, spares the superficial stratum, and merges laterally with interdigitating fasciculae of the brachium pontis (Wiel $\Omega$ $\times 4)$. 
spared the lateral lemniscus, medial longitudinal fasciculus, mesencephalic root, and motor nucleus of the trigeminal nerve. Laterally, there was sparing of the brachium pontis and cerebellar peduncles, while a narrow, non-demyelinated rim of the ventral superficial margin of the pons was intact. An isolated descending fasciculus of the cortico-spino-pontile tract was spared. The ventral boundary of demyelination was well demarcated, but the lateral borders were irregular, being delineated by tongues of relatively intact fibre bundles which penetrated the central area. Vacuolation of the myelin cylinders was noted at the margin (Fig. 8). Demylination appeared most advanced centrally where microcystic cavitation and neuroaxonal loss was also evident. More peripherally, the axons were intact in areas of extensive demyelination (Fig. 7) and the pontile nuclei were easily identified, although diminished in number. Such pontile neurones contained hyperchromatic nuclei and showed eosinophilic cytoplasmic homogenization. Many vacuolated, fat-filled 'gitter cells', with rare binucleate forms, were present throughout the pontine myelinolysis. Gliosis was minimal, but gemistocytic astrocytes and occasional elongated microglial forms were evident at the periphery. There was increased vascularity at the margin with endothelial cell hypertrophy and proliferation. There was no perivasular accentuation of the demyelination, but increased numbers of macrophages were noted locally in the periadventitial zones.

Similar more subacute demyelinative changes were present in the central portion of the corpus callosum (Fig. 4), middle cerebellar peduncles immediately lateral to the dentate nucleus, putamen, internal medullary lamina, fusiform gyrus (Fig. 5), and cerebellar medullary substance. In the cerebellum the demyelination extended into the white matter of the folia and was characterized by many fat-filled macrophagic cells with sparing of the axis cylinders (Fig. 6). Myelin vacuolation and digestive chambers of Cajal were in these foci, especially in the zone adjacent to the granular layer of the cerebellum. Elongated glial elements were more numerous here than in the pons.

\section{DISCUSSION}

This 48-year-old man presented a classical picture of acute and chronic relapsing pancreatitis of 15 years duration. During his last episode, his course was complicated by transient hyperlipaemia, hypocalcaemia, hyperglycaemia, dehydration and shock, transient jaundice, mild azotaemia, and akinetic mutism.

The hyperlipaemia was detected 20 hours after the onset of the abdominal pain, lasted four days, and was due primarily to increased triglyceride level with relatively less change noted in cholesterol. The nonfamilial nature of the hyperlipaemia in this case is suggested by the lack of xanthomatous stigmata and the rapid return to normal levels after amelioration of the pancreatitis. The relationship between lactescence and acute pancreatitis is obscure (Klatskin and Gordon, 1952). The case presented here unequivocally documents the occurrence of acute transient hyperlipaemia with acute alcoholic pancreatitis.

The syndrome of extreme hyperglycaemia, serum hyperosmolality, and dehydration in the absence of ketoacidosis has been delineated recently. Three cases have been reported in associated with acute pancreatitis (Ward, 1963; Bergoz and Hausser, 1964;
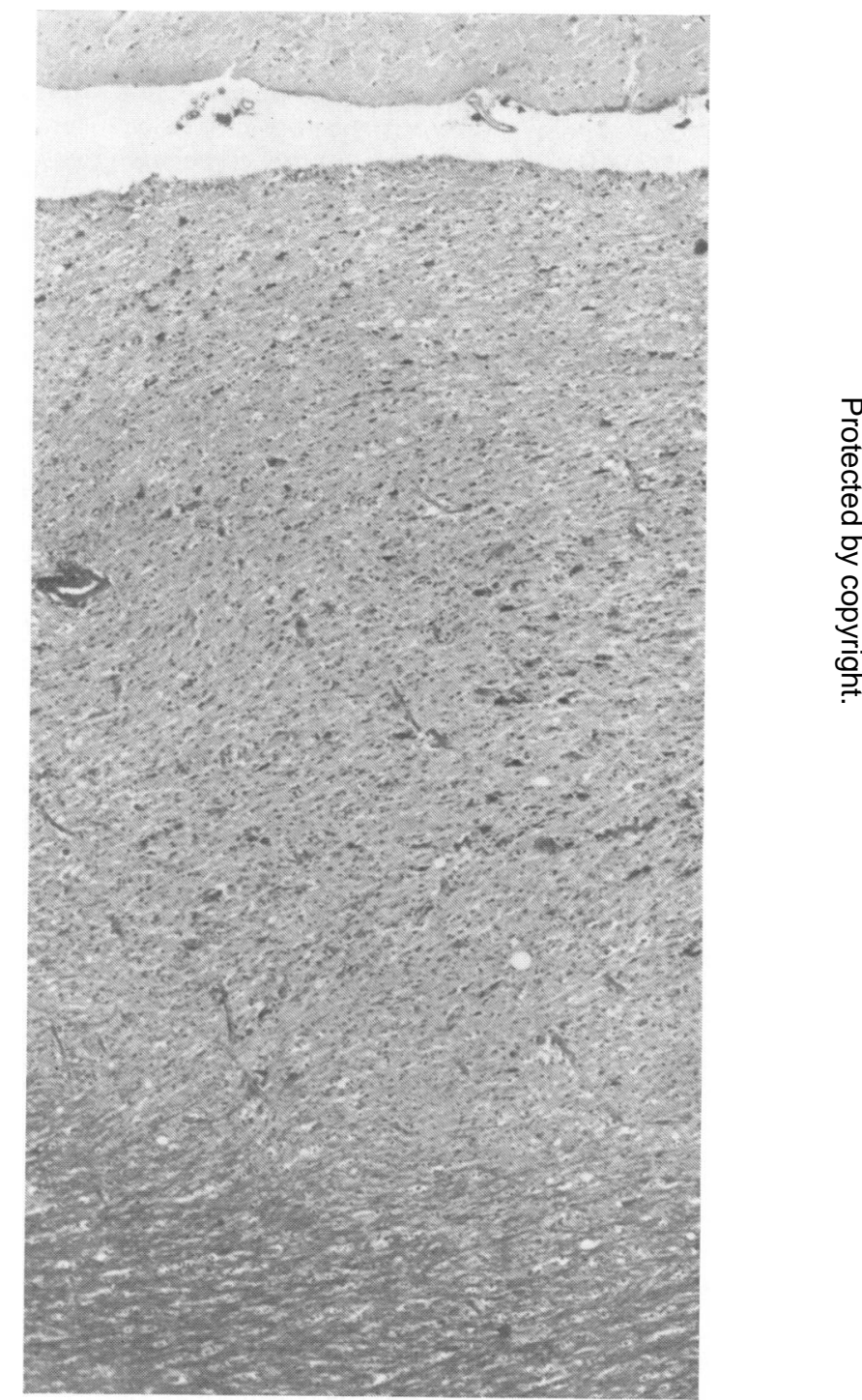

FIG. 4. Marginal zone of demyelination in the corpus callosum $($ Wiel $\times 40)$. 
Davidson, 1964). Flaccid or spastic paralysis, leading to profound coma, is usually a presenting finding in these patients. However, necropsy examinations failed to disclose any specific cerebral lesion, although authors have pointed to the severe neurological effects of hyperosmolality (Knowles, 1956). Calculations in our patient indicated a maximum hyperosmolality of approximately $416 \mathrm{~m}$-osmole/l. on the 12th day (Fig. 1).

Akinetic mutism is a clinical condition characterized by absolute mutism and clinical immobility except for the eyes. In a pathological study (Knowles, 1956), brain-stem infarction with central pontine involvement was present in seven out of eight cases. In the case presented there was predominant localization of demyelination in the pons, corpus callosum, and medullary substance of the cerebellum, with focal involvement of the middle cerebellar peduncles, putamen, and fusiform gyrus. There was no evidence of underlying vasculitis, inflammation, thrombosis, or fat embolism. Of these localizations, the most striking and perhaps clinically significant was the

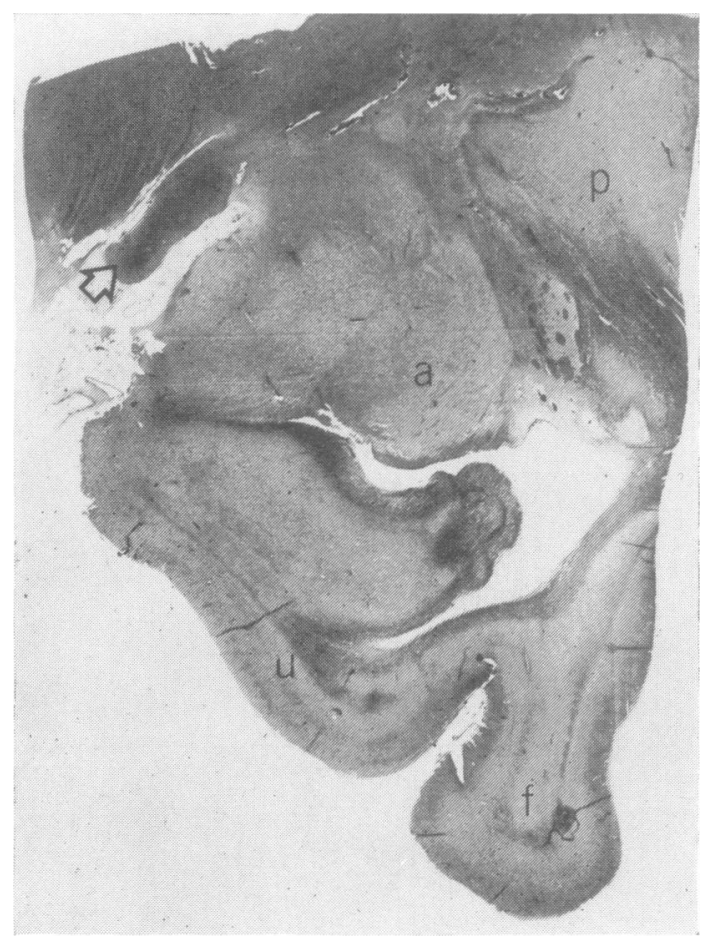

FIG. 5. Right coronal section through mesencephalon revealing optic tract (arrow), amygdaloid nucleus (a), putamen (p), uncal (u), and fusiform gyri (f). Note atrophy and demyelination of the fusiform gyrus with sparing of the uncinate fibres and demyelination of amygdala and anterior commissure $($ Wiel $\times 4)$. pontine lesion, which mirrored that of central $\underset{\mathbb{D}}{Z}$ pontine myelinolysis (Adams et al., 1959; Mathieson and Olszewski, 1960; Aleu and Terry, 1963; Berry of and Olszewski, 1963; Klavins, 1963; Behar, Bental, $\frac{c}{0}$ and Aviram, 1964; Kepes, Reece, and Oxley, 1965; Landers, Chason, and Samuel, 1965). Since first reported, this lesion has been the subject of numer- $\stackrel{\circ}{=}$. ous case reports, but the cause and pathogenesis of the demyelination remain speculative. A significant . number have shown extrapontine pathology, which $\vec{F}$ has included Wernicke's encephalopathy (four cases), $\frac{\overrightarrow{9}}{9}$ patchy cortico-neuronal loss and gliosis (six cases), pallidal gliosis and necrosis of the striatum (three $\frac{\bar{\sigma}}{\bar{\omega}}$ cases), and demyelination of the cerebellum in one $\frac{\sigma}{\sigma}$ case. Extra-pontine demyelination is striking in this $\varrho$ case with the associated involvement of the corpus $\%$ callosum, cerebellum, middle cerebellar peduncles $\vec{\circ}$ and putamen. Because of this dissemination, the possibility of an underlying vascular aetiology was $\vec{\omega}$ considered. The microscopic features of the demyelination are not consistent with an ischaemic necrosis because of preservation of neurones and absence of $\dot{\omega}$ subacute inflammation. Moreover, no venous or $\vec{\sigma}$ arterial occlusive disease is present in the basilat of

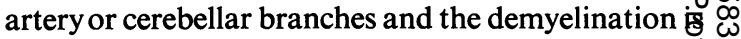
not perivascular in orientation. The symmetrical and $\mathrm{O}$ midline nature of the demyelination would argut equally against a vascular origin and an acute fug minating multiple sclerosis. Adams et al. (1959) have $\mathbb{\varnothing}$ cogently discussed this latter diagnosis with referenos to central pontine myelinolysis and have presented criteria for the pathological diagnosis of Schildere disease, post-infectious encephalomyelitis, and acue $\vec{\theta}$ necrotizing encephalitis. Perivascular demyelination 9 is a consistent feature of the above demyelinative encephalitides but is conspicuously absent in this case.

The case presented here is of unusual significance because of the accompanying demyelination of the $\mathbb{D}$ corpus callosum and, in this respect, is suggestive of $\overrightarrow{\vec{z}}$ Marchiafava-Bignami syndrome. Over 50 cases of this condition have been reported and the initial $\frac{\partial}{\overrightarrow{ }}$ emphasis given to Italian ancestry and ingestion of wine in the aetiology has been mitigated as a result of descriptions of cases in South Americans, French, Swiss, and English people without known trace of Italian ancestry. All cases had a history of under- 3 nutrition, alcoholism, or both. The disease is characterized by symmetrical degeneration of myelin $ᄋ$ most frequently and pathognomonically affecting the corpus callosum, with less frequent involvement of $\frac{9}{3}$ the anterior commissure, digital cerebral white $\vec{D}$ matter, and middle cerebellar peduncles. The symmetrical demyelination has a predilection for com- $N$ missural fibresystems. It is noteworthy that in none of the reported cases of Marchiafava-Bignami syn- $N$ 
drome has pontine demyelination been described. Conversely, demyelination of the corpus callosum has not been described in association with central pontine myelinolysis.

In the only case previously reported of cerebral demyelination associated with acute haemorrhagic pancreatitis (Vogel, 1951), the demyelination was irregular, non-symmetrical, predominantly localized to the cerebral hemispheres and basal ganglia, with no involvement of pons, corpus callosum or cere-

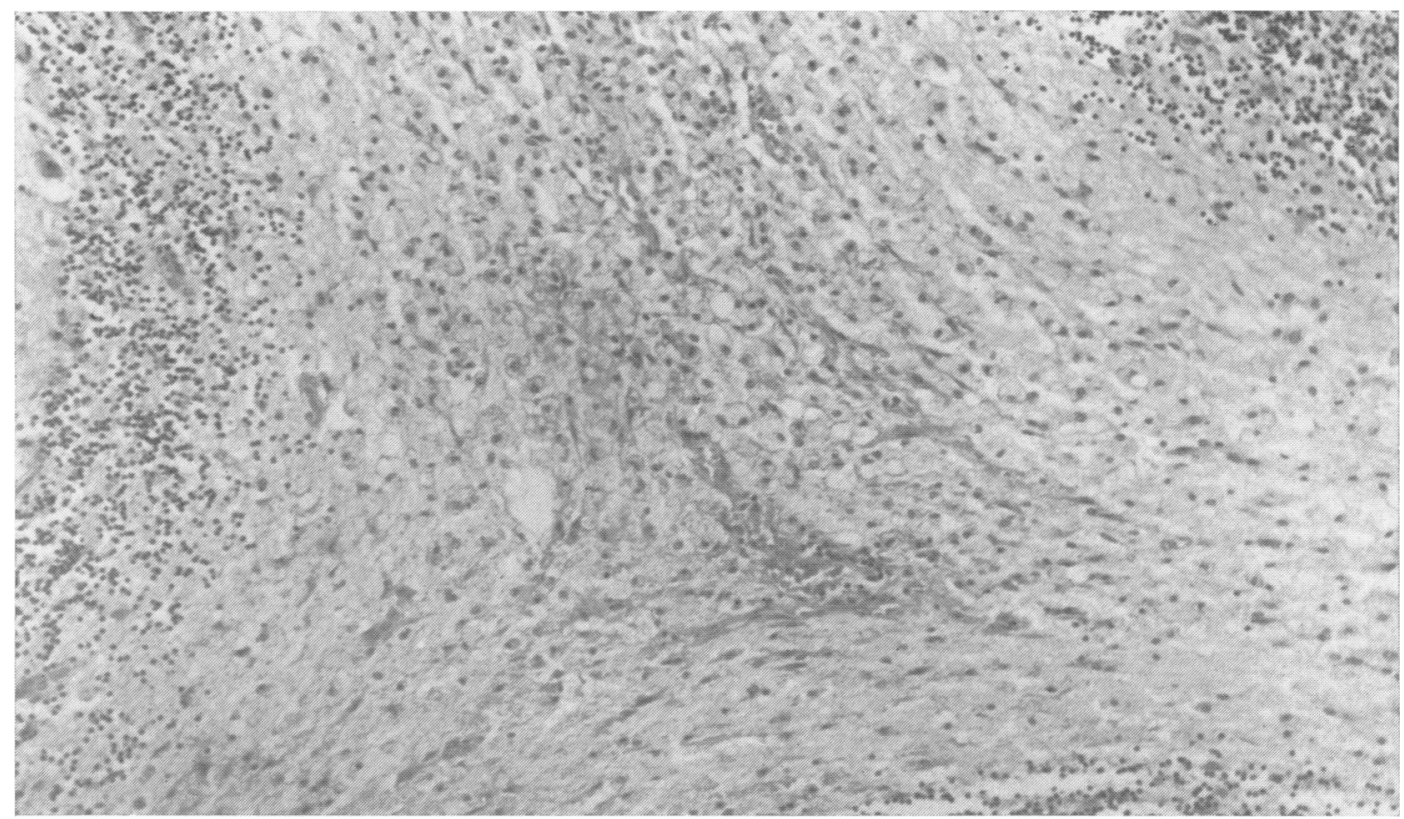

FIG. 6. Demyelination of medullary lamina of the cerebellum. Numerous macrophages, elongated microglial, and astroglial forms are noted $($ H.E. $\times 200)$.

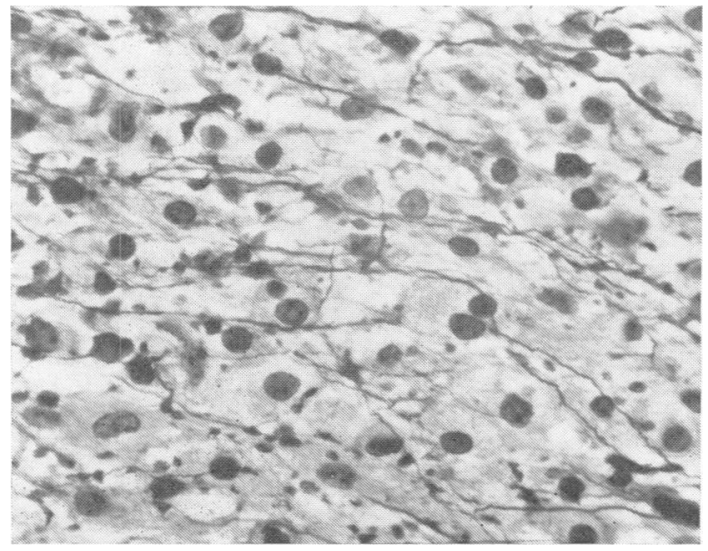

FIG. 7

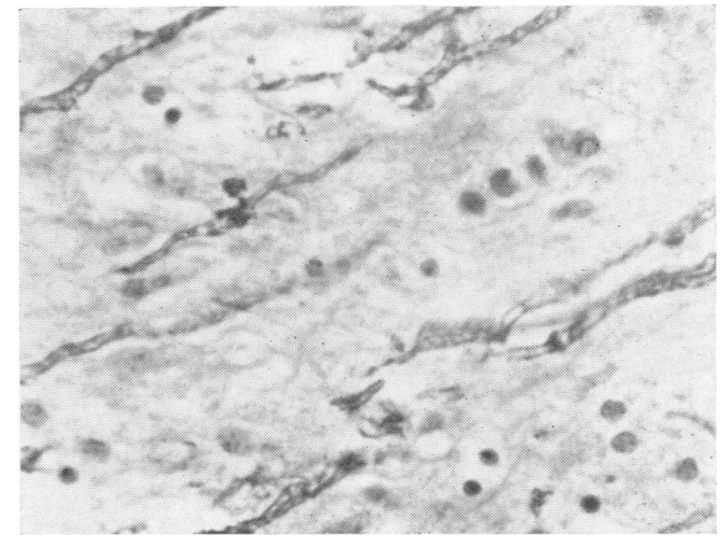

FIG. 8

FIG. 7. Preserved axons surrounded by numerous gitter cells running through area of pontine demyelination (Bodain $\times$ 950).

FIG. 8. Vacuolated segments of myelin and digestive chambers of Cajal at margins of cerebellar demyelination seen in Fig. 6. Identical changes were present at the lateral margins of pontine demyelination $($ Wiel $\times 950)$. 
bellum. Moreover, the lesions were present equally in grey and white matter but more extensive in the former. Of particular significance was the predominantly perivascular localization of the demyelination in contradistinction to the case here presented.

This case suggests a more fundamental cause for the demyelination. An outstanding clinical feature was the marked dehydration and hyperosmolality. The predominant demyelinative lesion appeared in the pons, presenting as central pontine myelinolysis. Arguments have been advanced that suggest alcoholism as the pathogenic mechanism for central pontine myelinolysis. Recent case reports (Bailey, Bruno, and Ober, 1960; Mathieson and Olszewski, 1960; Aki, Miyazakin, Takeuchi, Shimamine, and Aisawa, 1961; Kepes et al., 1965) among nonalcoholics have negated this simple explanation. Among non-alcoholics, some form of dehydration and electrolyte imbalance was present. The unique association of the Marchiafava-Bignami lesion and central pontine myelinolysis suggests that chronic alcoholism with impaired hepatic function (Shurtliff, Ajax, Englert, and D'Agostino, 1966) may play a role in the symmetrical cerebral demyelination seen here. However, severe fluid and electrolyte imbalance present in non-alcoholics with central pontine myelinolysis supports the hypothesis that severe hyperosmolality may also be an important aetiological factor. In the absence of venous thrombosis, we feel that demyelinative lesions may be initiated or accelerated in chronic alcoholism in the presence of acute, severe dehydration.

\section{SUMMARY}

A 48-year-old Negro male with a history of chronic relapsing alcoholic pancreatitis is presented. This man's illness was complicated by hyperlipaemia, hyperosmotic non-ketotic hyperglycaemic coma, akinetic mutism, cerebral and central pontine myelinolysis. The pathogenesis of these findings and their interrelationships are discussed. This case unequivocally documents the relationship of acute transient hyperlipaemia with alcoholic pancreatitis and appears to be the first reported association of the Marchiafava-Bignami syndrome and central pontine myelinolysis. Although representing the second documented case of cerebral demyelination following acute haemorrhagic pancreatitis, the nature of the demyelination in this case does not suggest a vascular aetiology.

We are grateful to Dean Sherman Mellinkoff for his constructive criticism in the preparation of this report.
This study was supported in part by Multiple Sclerosis Grant 385-1.

\section{ADDENDUM}

Our attention has been drawn to a paper by J. H. T) Adams emphasizing the importance of dehydration and electrolyte imbalance in two cases of non- $\frac{2}{2}$ alcoholic central pontine myelinolysis. Documentation of the serum biochemistry was includedAdams, J. H. (1962). Proceedings of the 4th International Congress of Neuropathology. Thieme, Stuttgart.

\section{REFERENCES}

Adams, J. H. (1962). Proceedings of the 4th International Congress of Neuropathology. Thieme, Stuttgart.

Adams, R. D., Victor, M., and Macall, E. L. (1959). Central pontine $\vec{O}$ myelinolysis, a hitherto undescribed disease occurring in alco- $\rightarrow$ holics and malnourished patients. Arch. Neurol. Psychiat. $\vec{\omega}$ (Chic.), 81, 154-172.

Aki, M., Miyazakin, M., Takeuchi, K., Shimamine, T., and Aisawa, S. (1961). Central pontine myelinolysis. Psychiat. Neurol. jap., 63, 408-413.

Aleu, F. P., and Terry, R. D. (1963). Central pontine myelinolysis. A $\dot{\omega}$ report of two cases. Arch. Path., 76, 140-146.

Bailey, O. T., Bruno, M. S., and Ober, W. B. (1960). Central pontine or myelinolysis. Amer. J. Med., 29, 902-906.

Behar, A., Bental, E., and Aviram, A. (1964). Central pontine myelin lysis. A case report. Acta. Neuropath. (Berl.), 3, 343-350.

Berry, K., and Olszewski, J. (1963). Central pontine myelinolysis. \$ case report. Neurology (Minneap.), 13, 531-537.

Bergoz, R., and Hauser, R. (1964). Diabetic coma without acido ketosis. Lancet, 1, 116.

Davidson, A. I. G. (1964). Diabetic coma without ketoacidosis in patient with acute pancreatitis. Brit. med. J., 1, 356.

Kepes, J. J., Reece, C. A., and Oxley, D. K. (1965). Central pontiñe myelinolysis in a 7-year-old boy.J. Neurol. Neurosurg. Psychiat 28, 39-47.

Klatskin, G., and Gordon, M. (1952). Relationship between relapsin pancreatitis and essential hyperlipemia. Amer. J. Med., 12, 3-23.

Klavins, J. V. (1963). Central pontine myelinolysis. J. Neuropath. exp. Neurol., 22, 302-317.

Knowles, H. C., Jr. (1956). Hypernatremia. Metabolism, 5, 508-518.

Landers, J. W., Chason, J. L., and Samuel, V. N. (1965). Central pontine myelinolysis, a pathogenic hypothesis. Neurology (Minneap.) 15, 968-971.

Lucas, P. F., and Owens, T. K. (1962). Subcutaneous fat necrosis, polyarthritis and pancreatic disease. Gut, 3, 146-148.

Lynch, M. J. (1954). Nephrosis and fat embolism in acute hemorrhagic pancreatitis. Arch. intern. Med., 94, 709-717.

Marchiafava, E., and Bignami, A. (1903). Sopra un'alterazione del corpo calloso osservata in soggetti alcoolisti. Riv. Pat. nerv. ment., 8, 544-549.

Mathieson, G., and Olszewski, J. (1960). Central pontine myelinolysis with other cerebral changes. A report of two cases. Neurology (Minneap.), 10, 345-354.

Shurtliff, L. F., Ajax, E. T., Englert, E. Jr., and D'Agostino, A. N. (1966). Central pontine myelinolysis and cirrhosis of the liver. Amer. J. clin. Path., 46, 239-244.

Swerdlow, A. B., Berman, M. E., Gibbel, M. I., and Valaitis, J. (1960) Subcutaneous fat necrosis associated with acute pancreatitis. $J$. Amer. Med. Ass., 173, 765-769.

Vogel, F. S. (1951). Cerebral demylination and focal lesions in case of acute hemorrhagic pancreatitis with consideration of possible role of circulating enzymes in the causation of the lesions. Arch. Path., 52, 355-63.

Ward, F. G. (1963). Diabetic coma without ketoacidosis. Lancet, 1, 450. 